\title{
Comparative Study of the Lipid Lowering Effect of Leaf Extract of Catharanthus roseus \& Atorvastatin
}

\author{
Sabina Jesmin ${ }^{1}$, Anisur Rahman Khan², Waseka Akther Jahan³, Md. Abdullah Yusuf ${ }^{4}$, \\ Wahida Begum 5 , Mahbuba Begum 6 , Showkat $\mathrm{Ara}^{7}$
}

\author{
${ }^{1}$ Assistant Professor, Department of Pharmacology, National Institute of Neuroscience \& Hospital, Dhaka, Bangladesh; \\ ${ }^{2}$ Assistant Professor, Department of Cardiology, Sir Salimullah Medical College \& Mitford Hospital, Dhaka, \\ Bangladesh; ${ }^{3}$ Assistant Professor, Department of Biochemistry, National Institute of Neuroscience \& Hospital, \\ Dhaka, Bangladesh; ${ }^{4}$ Assistant Professor, Department of Microbiology, National Institute of \\ Neuroscience \& Hospital, Dhaka, Bangladesh; ${ }^{5}$ Assistant Professor, Department of Radiology \& \\ Neuroimaging, National Institute of Neuroscience \& Hospital, Dhaka, Bangladesh; \\ ${ }^{6}$ Assistant Professor, Department of Surgery, Medical College for Women \& \\ Hospital, Dhaka, Bangladesh; ${ }^{7}$ Assistant Professor, Department of \\ Neuroradiology \& Imaging, National Institute of Neurosciences \& \\ Hospital, Dhaka, Bangladesh
}

[Received: January 2015; Revised: March 2015; Accepted: June 2015; Published: July 2015]

\begin{abstract}
Background: Leaf extract of Catharanthus roseus has medicinal effect. Objective: The purpose of the present study was to compare the effect of leaf extract of Catharanthus roseus (Nayantara) with atorvastatin on serum lipid profile in fat-fed rats. Methodology: This was an experimental animal study carried out in the Department of Pharmacology and Therapeutics at Dhaka Medical College, Dhaka from July 2005 to June 2006 for a period of one year. Adult Long Evans rats of both sexes were used for the study. The rats were divided into seven groups designed as A, B, C, D, E, F and G. Each group was comprised of 8 rats which were treated for 10 days and were sacrificed on 11th day. The experiment were designed to demonstrate the effect of C. roseus on serum lipid profile in hyperlipidaemic rats and the hypolipidaemic effects were compared to a standard oral lipid lowering drug, atorvastatin. Result: A total number of 56 adult Long Evans rats of both sexes were used for the study. Here the effect of leaf extract of C. roseus at different doses on lipid level of fat-fed rats was observed which were compared with the hyperlipidaemic control group. In the same time the effect of Atorvastatin on serum lipid level were observed. The observed lipid lowering effect of leaf extract of $\mathrm{C}$. roseus was evaluated biochemically by significant $(\mathrm{p}<0.001)$ decrease of serum total cholesterol (TCL), triglyceride (TG) and low density lipoprotein (LDL) levels. The leaf extract of $\mathrm{C}$. roseus was given at three different doses daily in oral route for 10 days along with fatty diet and statistically significant $(\mathrm{p}<0.05)$ decrease was observed in all serum lipid parameter $(\mathrm{TCL}, \mathrm{TG}$, LDL) except high density lipoprotein (HDL). Which was more evident in higher doses treated group (1 $\mathrm{ml} / \mathrm{kg}$ and $1.5 \mathrm{ml} / \mathrm{kg}$ ). Atorvastatin reduced all three parameter (TCL, TG, \&LDL) significantly $(\mathrm{p}<0.001)$. It increased HDL significantly high $(\mathrm{p}<0.001)$. Conclusion: The leaf extract of $\mathrm{C}$. roseus is responsible for lipid lowering effect. [Journal of National Institute of Neurosciences Bangladesh, 2015;1(2): 53-56]
\end{abstract}

Keywords: Catharanthus roseus; atorvastatin; hyperlipidaemic; hypolipidaemic

Correspondence: Dr. Sabina jesmin, Assistant Professor, Department of Pharmacology, National Institute of Neuroscience \& Hospital, Sher-E-Bangla Nagar, Agargaon, Dhaka-1207, Bangladesh; Email: sabinajesmin15@gmail.com; Cell no.: $+8801817066602$

Conflict of interest: There is no conflict of interest to any of the authors of this article.

Funding agency: The study was not funded by any authority.

Contribution to authors: SJ was involved in protocol preparation, data collection and literature search up to report writing. ARK, WAJ, MAY, WB, SA \& MB were involved in manuscript preparation as well as involved in literature search up and manuscript writing and manuscript revision.

How to cite this article: Jesmin S, Khan AR, Jahan WA, Yusuf MA, Begum W, Begum M, Ara S. Comparative Study of the Lipid Lowering Effect of Leaf Extract of Catharanthus-Roseus \& Atorvastatin. J Natl Inst Neurosci Bangladesh, 2015;1(2): 53-56 


\section{Introduction}

Metabolic disorders that involve elevation in any lipoprotein species are termed hyperlipoproteinemias or hyperlipidemias. Lipoprotein disorder or dyslipidemias are common metabolic disorder seen in clinical practice. The two major sequels of hyperlipidemias are pancreatitis and atherosclerosis; it may cause coronary heart diseases, dermatological manifestations (xanthelasmata \& xanthoma), neurological and ocular abnormalities ${ }^{1}$.

Coronary heart disease (CHD) is the commonest form of heart diseases and the single most important cause of premature death in the developed world ${ }^{2}$. As the incidence of CHD is increasing rapidly all over the world, the management of CHD is more targeted to prevention rather than treatment. Hyperlipidemia is one of the major risk factor for $\mathrm{CHD}^{3}$. So modification and treatment of the hyperlipidemia is one of the key factors of the prevention of CHD. Besides the conventional lipid-lowering agent, the use of herbal extract to lower the lipid level will open a new era in the field of management of hyperlipidemia.

Lipid research clinic's coronary primary prevention trial has provided useful information on the reduction of plasma cholesterol level in hyperlipidemic subjects by diet and drug therapy and thus, the reduction in risk of myocardial infarction and death ${ }^{2}$. However, drugs are costly and more toxic. Therefore, studies are still going on in search of more potent, less toxic, cheaper and easily available lipid lowering agents. Fulfilling these criteria's, many research works have been done on various herbal agents, such as garlic oil, onion, cowpea, karalla and many more. Therefore, the present study was undertaken to compare the effect of leaf extract of Catharanthus roseus (Nayantara) with atorvastatin on serum lipid profile in fat-fed rats.

\section{Methodology}

This was an animal study carried out in the Department of Pharmacology and Therapeutics at Dhaka Medical College, Dhaka from July 2005 to June 2006 for a period of one year. Long Evans rats of both sex and weighing 150 to 200 gram were selected for the present study. They were kept in animal house of the Department of Pharmacology and Therapeutics, Dhaka Medical College and were allowed to feed on standard laboratory diet and drink ad libitum. These rats were acclimatized for 5 days at room temperature and humidity. Fresh leaves of C. roseus were collected from the garden of Bangladesh council of scientific and industrial research (BCSIR), Dhaka. Crude extract for administration was prepared by crushing the leaves in a stainless steel mortar and then juice was separated by a fine cloth as a part of filtration. The juice was stored in refrigerator at $4^{\circ} \mathrm{C}$ until use for the experiment. Atorvastatin was used as standard lipid lowering agent and was collected from Alco-Pharma; furthermore, 1.5 $\mathrm{ml}$ olive oil plus $1.0 \%$ cholesterol daily orally was used as fatty diet. Ten (10) gram cholesterol was dissolved in $100 \mathrm{ml}$ olive oil; therefore, $1.5 \mathrm{ml}$ olive oil per rat (average weight 150) contained 0.15 gm cholesterol which was equivalent to $1 \%$ cholesterol diet ${ }^{5}$. Rats were divided into 7 groups each group contains 8 rats. For convenience, the experiments were divided into two parts; Ex-I \& Ex-II. Group A served as normal control group received normal diet $\&$ distilled water for 10 day. Group B hyperlipidemic experimental group received normal diet and $1.5 \mathrm{ml}$ olive oil with cholesterol per day for 10 day. Group A and group B comprises Experiment- I. Other 5 group include in experiment- II. Group C fat-fed control group received normal diet, distilled water and $1.5 \mathrm{ml}$ olive oil with cholesterol per day for 10 day. Group D, E, and F fat-fed experimental groups received normal diet, distilled water, $1.5 \mathrm{ml}$ olive oil with $1 \%$ cholesterol and $0.5 \mathrm{ml} / \mathrm{kg}, 1 \mathrm{ml} / \mathrm{kg}, 1.5 \mathrm{ml} / \mathrm{kg}$ leaf extract of C. roseus respectively daily for 10 days $^{3}$. And group $\mathrm{G}$ received normal diet, distilled water, $1.5 \mathrm{ml}$ olive oil with $1 \%$ cholesterol and $0.14 \mathrm{mg} / \mathrm{kg}$ atorvastatin for 10 day. On $11^{\text {th }}$ day of experiment, blood was collected by cardiac puncture and sera were obtained. Subsequently, serum TCL, TG, LDL and HDL levels were measured by enzymatic-colorimetric method in the Department of Clinical Pathology at Dhaka Medical College, Dhaka using lipid profile kits for estimation of serum lipid profile. The results obtained from the experiment are represented as mean \pm SEM of the number of samples. Data was analyzed by student's unpaired " $t$ " test and significant difference between means \pm SEM of the different groups were estimated using one way analysis of variance (ANOVA) followed by student's unpaired "t" test.

\section{Results}

In ex-I, regarding the lipid levels, it was observed that there was statistically significant rise of serum TCL, TG, LDL in group B that is hyperlipidemic experimental group. However, significant decrease of HDL level compared to group A (Table 1). In case of ex-2, it was observed that there was statistically significant rise of serum TCL, TG, LDL levels in hyperlipidemic control group $p<0.001$ in each parameter. Serum TCL, TG and LDL levels decrease in all C. roseus and atorvastatin treated group compared to the control group. Maximum effect was observed in 
atorvastatin \& higher doses of C. roseus treated group. Also LDL:HDL significantly decrease in these 2 group. However, no significant change was observed in low dose of C. roseus treated group (Table 2).

Table 1: SSerum Lipid Profile in Group A \& Group B Rats in Experiment-I

\begin{tabular}{lccc}
\hline \multirow{2}{*}{$\begin{array}{l}\text { Lipid Profile } \\
(\mathbf{m g} / \mathbf{d l})\end{array}$} & \multicolumn{2}{c}{ Group ( Mean \pm SEM) } & P value \\
\cline { 2 - 3 } & Group A & Group B & \\
\hline TCL & $79.63 \pm 0.86$ & $141.50 \pm 0.60$ & $<0.001^{* * *}$ \\
& $(75.0-82.0)$ & $(140.0-145.0)$ & \\
HDL & $33.25 \pm 1.00$ & $29.50 \pm 1.02$ & $<0.05^{*}$ \\
& $(30.0-37.0)$ & $(26.0-33.0)$ & \\
LDL & $15.13 \pm 0.74$ & $79.38 \pm 0.65$ & $<0.001^{* * *}$ \\
& $(12.0-17.0)$ & $(77.0-82.0)$ & \\
TG & $71.88 \pm 0.74$ & $108.13 \pm 1.20$ & $<0.001^{* * *}$ \\
& $(70.0-75.0)$ & $(105.0-115.0)$ & \\
\hline
\end{tabular}

$\mathrm{Ns}=$ not significant; $*=$ significant at $\mathrm{p}<0.05 ; * *=$ significant at $\mathrm{p}<0.01$; $* * *=$ significant at $p<0.001$

Table 2: Mean Lipid Profiles Of Different Groups of Rat in Experiment-II

\begin{tabular}{lcccc}
\hline Groups & \multicolumn{4}{c}{$\begin{array}{c}\text { Lipid Profiles (mg/dl) } \\
\text { (Mean } \pm \text { SEM) }\end{array}$} \\
\cline { 2 - 5 } & TCL & HDL & HDL & TG \\
\hline Group C & $142.63 \pm 0.65$ & $30.50 \pm 0.85$ & $30.50 \pm 0.85$ & $108.25 \pm 0.88$ \\
& $(140.0-145.0)$ & $(27.0-33.0)$ & $(27.0-33.0)$ & $(105.0-112.0)$ \\
Group D & $140.25 \pm 1.10$ & $28.88 \pm 0.55$ & $28.88 \pm 0.55$ & $103.00 \pm 0.89$ \\
& $(135.0-145.0)$ & $(27.0-31.0)$ & $(27.0-31.0)$ & $(100.0-107.0)$ \\
Group E & $136.25 \pm 1.37$ & $31.88 \pm 0.77$ & $31.88 \pm 0.77$ & $96.00 \pm 1.31$ \\
& $(130.0-140.0)$ & $(28.0-35.0)$ & $(28.0-35.0)$ & $(90.0-100.0)$ \\
Group F & $72.13 \pm 3.36$ & $30.50 \pm 0.73$ & $30.50 \pm 0.73$ & $95.00 \pm 1.30$ \\
& $(65.0-95.0)$ & $(28.0-34.0)$ & $(28.0-34.0)$ & $(90.0-100.0)$ \\
Group G & $63.00 \pm 1.24$ & $36.75 \pm 0.73$ & $36.75 \pm 0.73$ & $84.00 \pm 1.22$ \\
C VS D & $(60.0-70.0)$ & $(34.0-40.0)$ & $(34.0-40.0)$ & $(80.0-90.0)$ \\
C VS E & $>0.10 \mathrm{~ns}$ & $>0.10 \mathrm{~ns}$ & $>0.10 \mathrm{~ns}$ & $<0.001^{* * *}$ \\
C VS F & $<0.01^{* *}$ & $>0.10 \mathrm{~ns}$ & $>0.10 \mathrm{~ns}$ & $<0.001^{* * *}$ \\
C VS G & $<0.001^{* * *}$ & $>0.50 \mathrm{~ns}$ & $>0.50 \mathrm{~ns}$ & $<0.001^{* * *}$ \\
& $<0.001^{* * *}$ & $<0.001^{* * *}$ & $<0.001^{* * *}$ & $<0.001^{* * *}$ \\
\hline
\end{tabular}

$\mathrm{Ns}=$ not significant, $*=$ significant at $\mathrm{p}<0.05, * *=$ significant at $\mathrm{p}<0.01$, $* * *=$ significant at $\mathrm{p}<0.001$

\section{Discussion}

The present study was carried out to evaluate the effect of Catharanthus-roseus on serum lipid profile and was compared the effect with Atorvastatin. Hypolipidemic effects were tested on adult long Evans rats. In this work, hyperlipidemia was induced in rats by administration of $1.5 \mathrm{ml}$ olive oil with $1 \%$ cholesterol for 10 days. The hyperlipidemia was evidenced by a significant increase in serum TCL, TG and LDL levels. The serum HDL level significantly decreased. Similar observations were reported in different studies ${ }^{6-7}$ who had demonstrated hyperlipidemia by administering 1.5 ml olive oil solution containing vitamin D3 and cholesterol for 5 consecutive days.

In this study, concomitant administration of leaf extract of C. roseus and hyperlipidemic diet (olive oil + cholesterol) daily orally for 10 days reduced serum TCL, TG and LDL levels. The reduction was highly significant in compares to hyperlipidemic control group which was best observed at the higher dose like 1.0 and $1.5 \mathrm{ml} / \mathrm{kg} /$ day. However, the level of HDL did not alter by any of the doses studied; in one study, it was shown that the leaf juice of $\mathrm{C}$. roseus produced a significant decrease in serum TCL, TG, LDL and VLDL cholesterol in rats $^{3}$. The decreases were significant $(p<0.001)$ at the highest dose of the juice compared to control in case of TCL, LDL, TG and VLDL cholesterol. However, the level of HDL did not alter by any of the doses studied. The finding of the study is similar to the present study. The reduction of serum TCL, TG and LDL by higher doses of C. roseus $(1.0 \& 1.5 \mathrm{ml} / \mathrm{kg})$ were almost similar to that of Atorvastatin.

Though the exact mechanism of hypolipidemic effect of C. roseus is not yet known, it is believed that the reduction of the level of LDL and VLDL could have resulted from the antioxidant effect of the fresh leaf juice of C. roseus, whose phytochemical components include flavonoid, which is known for antioxidant effect ${ }^{4}$. However, no other work has been carried out on this aspect of $\mathrm{C}$. roseus in Bangladesh so far. Therefore, the result could not be correlated further with those of others. The present study provides an initial step in demonstrating the hypolipldemic effect of leaf extract of $\mathrm{C}$. roseus in hyperlipidemic state. The obtained data support the basis for future use of C. roseus in traditional system of medicine. Thus it could be a new agent in reducing morbidity and mortality resulting from dyslipidemia. However, the promising hypolipidemic effect of $\mathrm{C}$. roseus must be weighed along with its easy availability. The finding here provides a baseline for future studies designed to quantify the effects of leaf extract of C. roseus.

\section{Conclusion}

In conclusion the results and observations of the present study provide a rationale for use of C. roseus in the development of a new herbal medicine, much needed for the reduction of serum lipid levels (TCL, TG and LDL). Thus it could be useful in hyperlipidemic condition, such as atherosclerosis, obesity, hypertension, hyperlipidemia, IHD etc. But before establishing leaf extract of C. roseus as a therapeutically effective lipid lowering agent, further studies should be carried out to determine the active 
principles responsible for lipid lowering effect and its cellular mechanism of action. Toxicological studies in animal should also be undertaken as well before any clinical trial for suitability of using in man.

\section{References}

1. Edwards CRW, Endocrine and metabolic diseases. In: Edwards CRW, Bouchier I, Haslet C, Chilvers E, editors. Davidson's principles and practice of medicine. 19th ed. Edinburgh: Churchill Livingstone, 2002: 670-774

2. Hoeg JM, Richard E, Brewer HB. An approach to the manage- ment of hyperlipoproteinemia JAMA 1986; 255: 511-21

3. Anita BS, Okokon JE, Effect of leaf juice of Catharanthus roseus Linn. On cholesterol, Triglyceride, and lipoprotein levels in normal rats. Ind J pharmacol 2005; 37: 401-2

4. Ghani A. Medicinal plants of Bangladesh, 2003: 159-60

5. Akter A. Study of the effect of Neem oil on serum lipid profile in normal and fat fed rats (B.Sc. honours thesis). Dhaka: University of Dhaka, 1991

6. Lata S, Saxena KK, Bhasin V, Saxena RS, Kumar A, Srivastava VK. Beneficial effect of Allium sativa, Allium cepa and Commiphora mukul on experimental hyperlipidemia and atherosclerosis: A comparative evaluation. J Postgrad Med 1991; 132-5 